Z. Klin. Chem. Klin. Biochem.

13. Jg. 1975 , S. $423 \cdot-426$

\title{
Vergleichende Untersuchungen zur Bestimmung von Gesamtprotein und Albumin im Serum von Mensch, Affe, Hund und Ratte
}

\author{
Von A. Metz und Annerose Schütze
}

Medizinisch-Biologische Forschung Sandoz AG, Basel (Schweiz)

Zusammenfassung: Serumalbumin wird mit vier verschiedenen Farbreagenzien (Proteinbindungsreagenzien) bei Mensch und Tier bestimmt. Die Resultate werden mit den entsprechenden Ergebnissen aus Elektrophorese und Biuretmethode verglichen, wobei Versatol als Standardserum dient. Bei der Albuminbestimmung mit 2-(4-Hydroxyazobenzol)-benzoesäure- und Bromkresolpurpur-Reagenz zeigen Affe (Rhesus), Hund (Beagle) und Ratte deutliche Bindungsdifferenzen, während die Bromkresolgrünmethode solche Differenzen nur bei der Ratte aufweist.

Die Albuminkonzentration ist bei Mensch, Affe und Ratte gleich hoch, der Totalproteingehalt hingegen nur bei Mensch und Affe.

\section{A comparative study of total protein and albumin in man, monkey, dog and rat employing different analytical methods}

Summary: In the present study, serum albumin was determined colorimetrically in three animal species and in man with the help of four currently used dye-reagents (protein binding reagents). The results were compared with those obtained from corresponding electrophoretic and biuret determinations, using Versatol as a standard-control throughout. Using 2-(4-hydroxyazobenzene)-benzoic acid and bromocresol purple as reagent for the albumin determinations, species-specific differences from the electrophoretic results were found. No such differences occurred with bromocresol green, except in rats.

There was no significant difference in albumin concentrations between man, monkeys and rats. Total protein concentration was only similar in man and monkeys.

\section{Einfuihrung}

Für die Routinebestimmung von Serumalbumin stehen uns heute im wesentlichen zwei Möglichkeiten zur Verfügung, Elektrophorese und kolorimetrische Verfahren.

Letztere bedienen sich der Tatsache, daß Azofarbstoffe wie z. B. 2-(4-Hydroxyazobenzol)-benzoesäure Bromkresolgrïn, Bromkresolpurpur, Methylorange, Methylrot und Spectu AB-2(1) mit Albumin, entsprechend dessen Bindungskapazität für diese Stoffe, Albumin-Azofarbstoffkomplexe bilden, die photometrisch bestimmt werden können. Von diesen Farbstoffen hat in der Vergangenheit 2-(4-Hydroxyazobenzol)-benzoesäure (2-5) als Albuminreagenz die verbreitetste Anwendung gefunden. Dies mag natürlich einer der Gründe sein, daß man auch seine Nachteile am besten kennt: schlechte Korrelation mit anderen Methoden, Interferenzen mit Bilirubin, Fettsäuren und Arzneimitteln, wie Salicylaten, Sulfonamiden und Penicillin (5-7), sowie „Speziesunterschiede“ (8-9). All diese Erkenntnisse waren gerade in letzter Zeit für verschiedene Autoren Anlaß, die Bromkresolgrün-Methode (1, 7, 10, $11,12)$ und die Bestimmung mit Bromkresolpurpur (13) zu empfehlen.
Über die routinemäßige Verwendbarkeit letzterer Methoden bei Laboratoriumstieren ist unseres Wissens in der einschlägigen Literatur nichts beschrieben. Ziel vorliegender Arbeit ist es deshalb, diese bestehende Lücke zu schließen, aus Gründen der Qualitätskontrolle die wechselseitige Verwendung von Tier- und Humanserum zu prüfen, Speziesdifferenzen aufzuzeigen und die fựr das Tierlaboratorium günstigste Methode herauszufinden. Auch steht im Hintergrund die Problematik der Verwendung eines einheitlichen Standards bei verschiedenen Spezies im Rahmen multipler Analysenverfahren. Auf mögliche Interferenzen mit Arzneimitteln soll im Rahmen dieser Arbeit hingewiesen, jedoch in praktischen Versuchen nicht eingegangen werden.

Es werden die vier bekanntesten Verfahren der Albuminbestimmung, Elektrophorese, 2-(4-Hydroxyazobenzol)-benzoesäure-Verfahren (mit und ohne Zusatz von Detergenzien), Bromkresolgrün- und BromkresolpurpurMethode, sowie die Proteinbestimmung mit Biuretreagenz, vergleichend bei verschiedenen Spezies untersucht. Im nachfolgenden Text soll anstelle der ausgeschriebenen Bezeichnungen die in Literatur und Routine gebräuchlichen Abkürzungen verwendet werden: 
Tab. 1. Methodenvergleich zwischen Elektrophorese und photometrischen Albuminbestimmungen mittels HABA-, BCG- und BCPReagenzien bei Mensch, Affe, Hund und Ratte (Mittelwerte von $5 \delta$ und 59 pro Spezies \pm Standardabweichung)

\begin{tabular}{|c|c|c|c|c|c|c|c|}
\hline Spezies & Totalprotein & Albumin (g/l) & & & & & \\
\hline & $(g / l)$ & $\begin{array}{l}\text { Elektro- } \\
\text { phorese }\end{array}$ & $\begin{array}{l}\text { HABA (ohne } \\
\text { Detergenz) }\end{array}$ & $\begin{array}{l}\text { HABA } \\
\text { (mit Brij) }\end{array}$ & $\begin{array}{l}\text { HABA (mit } \\
\text { Levor IV) }\end{array}$ & $\begin{array}{l}\text { Bromkresol- } \\
\text { grün }^{\oplus}\end{array}$ & $\begin{array}{l}\text { Bromkresol- } \\
\text { purpur }^{\oplus}\end{array}$ \\
\hline Ratte & $64 \pm 4$ & $46 \pm 3$ & $\begin{array}{l}20 \pm 2 \\
* * *\end{array}$ & $\begin{array}{l}14 \pm 2 \\
* * *\end{array}$ & $\begin{array}{l}26 \pm 3 \\
* * *\end{array}$ & $\begin{array}{l}32 \pm 3 \\
* * *\end{array}$ & $\begin{array}{l}11 \pm 2 \\
* * *\end{array}$ \\
\hline Hund & $58 \pm 2$ & $31 \pm 3$ & $\begin{array}{l}17 \pm 2 \\
* * *\end{array}$ & $\begin{array}{l}15 \pm 2 \\
* * *\end{array}$ & $\begin{array}{l}31 \pm 4 \\
-\end{array}$ & $\begin{array}{l}32 \pm 2 \\
-\end{array}$ & $\underset{* * *}{8 \pm 1}$ \\
\hline Affe & $74 \pm 3$ & $47 \pm 4$ & $\begin{array}{l}27 \pm 2 \\
* * *\end{array}$ & $\begin{array}{l}37 \pm 4 \\
* * *\end{array}$ & $\begin{array}{l}42 \pm 2 \\
* *\end{array}$ & $\begin{array}{l}47 \pm 3 \\
-\end{array}$ & $\begin{array}{l}38 \pm 4 \\
* * *\end{array}$ \\
\hline Mensch & $71 \pm 3$ & $44 \pm 4$ & $\begin{array}{l}40 \pm 3 \\
*\end{array}$ & $\begin{array}{l}45 \pm 3 \\
-\end{array}$ & $\begin{array}{l}45 \pm 3 \\
-\end{array}$ & $\begin{array}{l}46 \pm 5 \\
-\end{array}$ & $\begin{array}{l}53 \pm 7 \\
* *\end{array}$ \\
\hline
\end{tabular}

*: Signifikanz der Unterschiede zwischen photometrischer Albuminbestimmung und Elektrophorese (gleiche Spezies).

(* $\mathrm{p}<0,05 ; * * \mathrm{p}<0,01 ; * * * \mathrm{p}<0,001$;

$\oplus$ : Detergenzzusatz nach Vorschrift.

HABA für 2-(4-Hydroxyazobenzol)-benzoesäure, BCG für Bromkresolgrün und BCP für Bromkresolpurpur.

\section{Material und Methoden}

\section{Apparaturen}

Blutzentrifuge (MSE); Technicon-Autoanalyzersystem mit eingebautem Eppendorf-Photometer (Einkanalsystem); Mikrophoresesy stem (Cellogel als Träger); W+W-Schreiber 4201 mit Integrator (Dr. Vaudaux, Basel); Zeiss-Scanner M4 QII.

\section{Reagenzien}

HABA-Dye (6 mmol/1) T 21-0179-07 (Technicon Genf); Formaldehyd (3999, $370 \mathrm{~g} / \mathrm{kg}^{1}$ ); Eisessig (90063); Natriumacetat (6267); Natriumhydroxid (6498); Kaliumjodid (5043); Kaliumnatriumtartrat (8087); Kupfersulfat (2791); Bromkresolgrün (8121); Bromkresolpurpur (3025); Brij-35 (Atlas Chemie, Essen); Methanol (6009); 5,5-Diäthylbarbitursäure (Na-Salz) (6318); Ponceau S (Fluka 81460); Barbital (276); Levor IV (Technicon Genf).

Methoden

Totalprotein wurde mit Biuretreagenz (14), Albumin mit HABA- (4) (mit und ohne die Detergenzien Brij und Levor IV), mit Bromkresolgrün- (12) und mit Bromkresolpurpurreagenz (13), nach den Angaben der Literatur, am Autoanalyzer bestimmt. Die elektrophoretische Auftrennung von Serumeiweiß für die Albuminbestimmung wurde auf Cellogel bei 230 Volt durchgeführt. Bei Mensch, Affe und Hund betrug die Laufzeit 21 Minuten; bei der Ratte mußte diese zwecks besserer Auftrennung auf 30 Minuten verlängert werden. Die bei der Elektrophorese verwendete Pufferlösung enthielt im Liter 7,28 $\mathrm{mmol}$ Diäthylbarbitursäure und 49,5 mmol Na-Diäthylbarbiturat (pH 8,6); Färbelösung (Ponceau S), Entfärbe- und Transparentlösung wurden nach der Vorschrift der Firma Chemetron Milano (für Cellogel) hergestellt. Alle statistischen Berechnungen wurden mit dem t-Test nach Student durchgeführt.

\section{Blutaufbereitung}

Die Blutentnahme erfolgte je nach Eigenart der Spezies

( 5 o und 5\%): beim Menschen (gesunde Probanden, 20-40 Jahre) aus den Vena cubitalis, beim Affen (indische Rhesusaffen,

1 Wenn nicht anders vermerkt, wurden Reagenzien p. a. der Firma Merck Darmstadt verwendet. etwa 2-3 Jahre alt) aus der Vena saphena magna, beim Hund (Beagles, 6-7 Monate alt) aus der Vena brachialis und bei SPF-Ratten (OFA-Sandoz, 8 Wochen alt) retroorbital. Das Serum wurde durch 5 min Zentrifugieren bei $4000 \mathrm{~g}$ gewonnen.

Standard- und Kontrollserum

Als Standard-bzw. Kontrollserum wurde Versatol der gleichen Charge (Cosmopharm Zürich) verwendet, auf welchem auch alle Berechnungen von Protein- und Albuminwerten basieren. Dic Resultate wurden mit Hilfe von Standardkurven ermittelt (vier Standards mit linear steigender Konzentration). Es wurden Doppelbestimmungen und daneben ,Absolutmessungen “ in Form von Extinktionsmessungen durchgeführt.

\section{Ergebnisse}

Die auf Versatol als Standard basierenden photometrischen Ergebnisse der Albuminbestimmung bei vier Spezies sind in Tabelle 1 mit den Resultaten aus Elektrophorese und Totalproteinbestimmung verglichen. Unter den hier gewählten Standardbedingungen führen beim Menschen nur die detergenzhaltigen HABA- und BCGReagenzien zu gleichen Albuminwerten wie die Elektrophorese. Beim Hund stimmen die Ergebnisse der HABA-Methode (in Anwesenheit von Levor IV) und die des BCG-Verfahrens mit den elektrophoretischen Resultaten überein; beim Affen zeigen sich nur mit BCG-Reagenz die gleichen Serumkonzentrationen wie bei der Elektrophorese. Nicht übereinstimmende Werte liefern sämtliche photometrischen Bestimmungen bei der Ratte. (Bemerkenswert bei diesem Tier ist die schon erwähnte geringere Wạnderungsgeschwindigkeit bei der elektrophoretischen Trennung von Albumin unter den mit den übrigen Spezies vergleichbaren Bedingungen). Von der Elektrophorese völlig abweichende Werte zeigt bei allen untersuchten Spezies die Bestimmung mit BCP-Reagenz.

Um auszuschließen, daß es sich um Relativdifferenzen (z. B. zum Standard) handeln könnte, hạben wir an zehn Tieren pro Spezies, neben den Autoanalyzerverfahren, manuelle Mesșungen mit steigenden Mengen Serum 

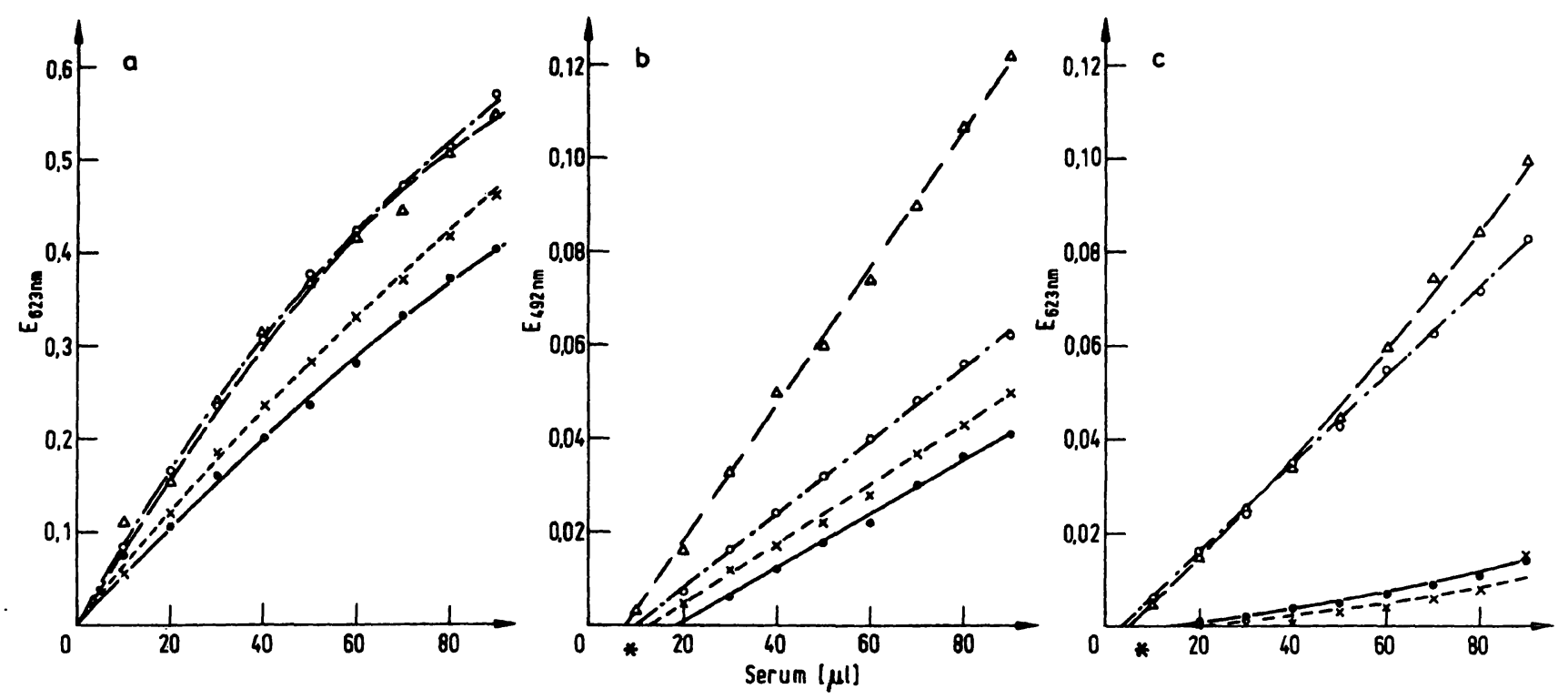

Abb. 1. Abhängigkeit der Extinktion von der Albuminkonzentration (ausgedrückt in $\mu \mathrm{l} \mathrm{Serum} / 5 \mathrm{ml}$ Testansatz: 10, 20, 30, 40, 50, $60,70,80,90 \mu 1 / 5 \mathrm{ml}$ ). Die Messungen wurden aus einem Scrumpool von 10 Individuen/Spczies durchgeführt.

a) Bromkresolgrün-Methode

b) 2-(4-Hydroxyazobenzol)-benzoesäureMethode

$\longrightarrow$ Hund

$\circ$ - $\circ$ Affe

$\Delta-\Delta$ Mensch

* Grenze der genauen Ablesbarkeit

$(10,20,30,40,50,60,70,80,90 \mu \mathrm{l} / 5 \mathrm{ml}$ Reagenzlösung) durchgeführt und die Extinktion gemessen. Die Absorptionskurven (Abb. 1) zeigen die gleichen Speziesunterschiede wie die auf Versatol basierenden Resultate. Gleichzeitig kann aus dieser Darstellung der Linearitätsbereich, die Wiederfindungsrate und die Reproduzierbarkeit der Methode für die manuelle sowie für die mechanisierte Analyse herausgelesen werden.

Tab. 2. Vergleichsstudie mit Werten der Elektrophorese (Eph) und BCG bei Ratten ( $\delta / \%=1: 1)$ zur Ermittlung eines mittleren Fehlerquotienten (F) bzw. Faktors

\begin{tabular}{|c|c|c|c|}
\hline \multirow[t]{2}{*}{$n$} & \multicolumn{2}{|l|}{ Albumin $(g / 1)$} & \multirow{2}{*}{$\begin{array}{l}\text { Quotient } \\
\text { Eph/BCG } \\
\overline{\mathrm{F}} \pm \Delta \mathrm{F}\end{array}$} \\
\hline & $\begin{array}{l}\text { Elektrophorese* } \\
\bar{x} \pm s\end{array}$ & $\begin{array}{l}\mathrm{BCG} * \\
\bar{x} \pm s\end{array}$ & \\
\hline $\begin{array}{l}30 \\
20 \\
20 \\
20 \\
10\end{array}$ & $\begin{array}{l}44,4 \pm 3,3 \\
48,6 \pm 3,7 \\
49,1 \pm 3,9 \\
46,1 \pm 4,6 \\
45,6 \pm 2,7\end{array}$ & $\begin{array}{l}31,4 \pm 2,9 \\
31,2 \pm 1,8 \\
32,1 \pm 2,0 \\
31,4 \pm 1,6 \\
34,4 \pm 2,5\end{array}$ & $\begin{array}{l}1,42 \pm 0,13 \\
1,56 \pm 0,15 \\
1,54 \pm 0,13 \\
1,48 \pm 0,17 \\
1,33 \pm 0,11\end{array}$ \\
\hline 100 & $46,7 \pm 4,1$ & $31,8 \pm 2,4$ & $1,47 \pm 0,16$ \\
\hline
\end{tabular}

\section{n: $\quad$ Anzahl der Tiere}

*: $\quad$ in beiden Fällen liegt eine Normalverteilung der Werte vor (ber. aus 100 Werten versch. Tiere)

$\overline{\mathrm{x}} \pm \mathrm{s}:$ Mittelwert + Standardabweichung; $\mathrm{F} \pm \Delta \mathrm{F}$ : Mittlerer Fehlerquotient \pm Standardabweichung.

Die Errechnung des Quotienten Eph/BCG erfolgte aus den Einzelwerten von füf verschiedenen Serieuntersuchungen, wobei die mittleren Fehlerquotienten der einzelnen Serien, sówie der aus allen Einzelwerten errechnete Wert, angegeben wird. Die Untersuchungen wurden an verschiedenen Tagen durchgefuhrt.
Die Ergebnisse bei Ratten veranlaßten uns zu weiteren Vergleichsstudien zwischen BCG und Elektrophorese. Es sollte untersucht werden, ob dieser Unterschied in den Ergebnissen zwischen Elektrophorese und BCGMethode sich auch bei größeren, verschiedenen Kollektiven reproduzieren läßt. Die Untersuchungsergebnisse in Tabelle 2 zeigen, daß bei Ratten das Verhältnis der Resultate Elektrophorese zu BCG relativ konstant bleibt $(1,47 \pm 0,16)$.

Die in Tabelle 1 auftretenden Speziesunterschiede im Totalprotein- und Albumingehalt veranlaßten uns, diese Differenzen statistisch zu überprüfen. Mit Hilfe des tTestes wurden die Konzentrationsunterschiede von Spezies zu Spezies getestet. Die mittleren Differenzen sind in Tabelle 3 dargestellt. Danach ist der Albumingehalt bei den Spezies Mensch, Affe und Ratte nicht signifikant voneinander verschieden. Bei Totalprotein andererseits zeigen nur Mensch und Affe vergleichbare Konzentrationen.

\section{Diskussion}

Automatisierung, Standardisierung und Qualitätskontrolle zwingen den Analytiker heute, sich wieder mehr mit methodischen Grundproblemen auseinander zu setzen. Dies trifft um so mehr zu, wenn man durch den Einsatz multipler Analyzersysteme mit einheitlichem Standard- bzw. Kontrollserum arbeiten will. Anstelle des für Bestimmungen an Menschen speziell empfohlenen Albuminstandards $(5,6,13)$ haben wir aus eingangs erwähnten Gründen die Verwendbarkeit 
Tab. 3. Statistische Untersuchungen auf Interspeziesdifferenzen von Totalprotein und Albumin bei Mensch, Affe, Hund und Ratte ( $t$-Test).

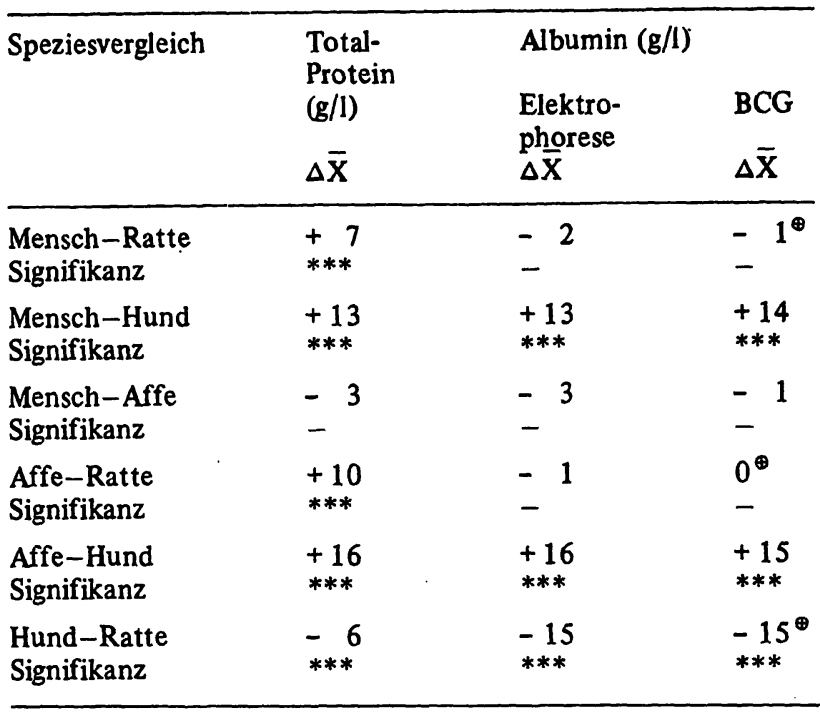

$\Delta \overline{\mathrm{X}}: \quad$ Speziesunterschiede in der Eiweiß- bzw. Albuminkonzentration.

$\oplus$ : bei Ratten, berechnet unter Verwendung des Fehlerquotienten

Signifikanz der Speziesunterschiede:

$*: \mathrm{p}<0,05,{ }^{* *}: \mathrm{p}<0,01,{ }^{* * *}: \mathrm{p}<0,001$.

eines Humanserums (Versatol) als Standard für die Albuminbestimmung bei verschiedenen Spezies getestet. Seine allgemeine Anwendbarkeit wird je nach Methode durch verschieden stark auftretende Proteinbindungsspeziesdifferenzen eingeschränkt. Dabei kennzeichnet sich die Bestimmung mit BromkresolgrünReagenz als das universell anwendbare Verfahren mit den geringsten Speziesunterschieden (Ausnahme Ratte); dies wurde bereits bestätigt durch ähnliche Ergebnisse bei Schwein, Rind, Pferd, Kaninchen und Schaf (1). Allgemein aber darf gesagt werden, daß die Albuminbestimmung auf der Basis von Protein-Farbstoffkomplexen in der Beurteilung ihrer Ergebnisse problematisch werden kann und daher besonders in Zweifelsfällen auf spezifischere Methoden zurückgegriffen werden sollte (z. B. immunologische Bestimmungen, sofern. die entsprechenden Voraussetzungen gegeben sind); denn wenn; ähnlich wie bei gewissen Arzneimitteln, die Anwesenheit mehrerer Stoffe im Blut sowohl eine gegenseitige Verschiebung aus der Eiweißbindung, als auch eine gegenseitige Verstärkung der Proteinbindung zur Folge haben kann (15), sollte auch im Fall der Albuminerfassung auf Proteinbindungsbasis an ähnliche Interferenzmöglichkeiten gedacht werden. Die in Tabelle 1 dargestellten Unterschiede in den Ergebnissen der Albuminwerte mit und ohne Zusatz von Detergenzien sprechen im Fall der Albuminbestimmung mit HABA für ähnliche Hemm- und Verstärkungsmöglichkeiten in der Proteinbindung. Sie könnten möglicherweise auch von substanz- bzw. detergenzbedingten Änderungen in der Sekundärstruktur des Albuminmoleküls herrühren. $\mathrm{Ob}$ und wie weit andererseits jedoch Zusätze von Stoffen bzw. gewissen Detergenzien substanz- und speziesbedingte Bindungsinterferenzen generell auszuschalten vermögen, muß Ziel weiterer umfangreicher Untersuchungen bleiben. Die Unterschiede im Totalprotein- und Albumingehalt, sowie die Unterschiede in der Proteinbindung der hier verwendeten, relativ jungen, jedoch geschlechtsreifen Tiere decken sich mit den von uns seit Jahren in der Routine gemachten Beobachtungen.

\section{Literatur}

1. Pemberton, J. R. \& De Jong, J. (1971), Analyt. Biochem. 43, 575-581.

2. Rutstein, D. D., Ingenito, E. F. \& Reynolds, W. E. (1953), J. Clin. Invest. 33, 211-220.

3. Wrenn, R. T. \& Feichtmeir, T. V. (1956), Tech. Bull. Reg. Med: Tech. 26, 174 .

4. Stevens, D. L. Technicon-Methodologie N-15b.

5. Ness, A. T., Dickerson, H. C. \& Pastewka, J. V. (1965), Clin. Chim. Acta 12, 532-541.

6. Arvan, D. A. \& Ritz, A. (1969), Clin. Chim. Acta 26, 505-516.

7. Leonard, P. J., Persaud, J. \& Motwani, R. (1971), Clin. Chim. Acta 35, 409-412.

8. Baxter, J. H. (1964), Arch. Biochem. Biophys. 108, 375-383.

9. Witiak, D. T. \& Whitehouse, M. W. (1968), Biochem. Pharmacol. 18, 971-977.

10. Doumas, B. T., Watson, W. A. \& Biggs, H. G. (1971), Clin. Chim. Acta 31, 87-96.

11. Beng, C. G. \& Lim, K. L. (1973), Amer. J. Clin. Path. 59, 14-21.

12. Schirardin, H. \& Nẹy, J. (1972), diese Z. 10, 338-344.

13. Ripoll, J. P. \& Colobert, L. (1971), Sym. Int. Technicon, Paris.

14. Weichselbaum, T. E., Technicon-Autoanalyzer-Methodologie N-14b.

15. Kriegelstein, J., Franz, J. W. \& Jähnchen, E. (1969), Naunyn-Schmiedeberg's Arch. Pharm. 264, 261.

Dr. A. Metz SANDOZ AG Pharm. Dept. CH-4002 Basel 